\title{
Direccionamiento Estratégico: Proyección de la Innovación Tecnológica y Gestión Administrativa en las Pequeñas Empresas
}

\author{
Hugo G. Hernández ${ }^{(1) \star}$, Diego A. Cardona ${ }^{(2)}$ y Jorge L. Del Rio ${ }^{(3)}$ \\ (1) Corporación Universitaria Latinoamericana (CUL), Barranquilla-Colombia \\ (e-mail: hugoghernandezpalma@gmail.com. \\ (2) Universidad Libre, Cartagena-Colombia (e-mail: diego.cardona@unilibrectg.edu.co) \\ (3) Universidad Tecnológica de Bolívar, Cartagena-Colombia (e-mail: jdelrio@unitecnologica.edu.co)
}

*Autor a quien debe ser enviada la correspondencia

Recibido Mar. 7, 2017; Aceptado May. 8, 2017; Versión final Jun. 28, 2017, Publicado Oct. 2017

\begin{abstract}
Resumen
El objetivo principal de esta investigación, es establecer el nivel de innovación en tecnología y direccionamiento estratégico de las pequeñas y medianas empresas (Pymes) de la ciudad de Barranquilla, Colombia. Para tal fin, se realizó una investigación de carácter descriptivo, mediante una técnica de recolección de datos, en la que se analizaron seis variables que determinaron el tamaño de la empresa en cuanto a número de trabajadores, el tipo de actividades innovadoras que implementan, el presupuesto que invierten en tecnología, las preferencias en cuanto al ámbito en el que innovan y las relaciones existentes entre lo que invierten y las innovaciones tecnológicas llevadas a cabo en una muestra de diez empresas de la ciudad. Los resultados de este ejercicio, demuestran que más de la mitad de las empresas pertenecientes a la muestra son medianas empresas, y solo un $40 \%$ de ellas desarrollan actividades enfocadas a la innovación tecnológica, preferiblemente que involucren acciones de comercialización y diseño, invirtiendo cantidades de recursos que se reflejan en el desarrollo organizacional.
\end{abstract}

\section{Strategic Direction: Projection of Technological Innovation and Administrative Management in Small Enterprises}

\begin{abstract}
The main objective of this research is to establish the level of innovation in technology and strategic direction of small and medium size enterprises (SMEs) in the city of Barranquilla, Colombia. For this purpose, a descriptive research was carried out, using a data collection technique, which analyzed six variables that determined the size of the company in terms of number of workers, the type of innovative activities they implement, the budget that invest in technology, the preferences in the area in which they innovate and the existing relationships between what they invest and the technological innovations carried out in a sample of ten companies of the city. The results of this exercise show that more than half of the companies belonging to the sample are medium-sized enterprises, and only $40 \%$ of them develop activities focused on technological innovation, preferably involving marketing and design actions, investing quantities that are reflected in the organizational development.
\end{abstract}

Keywords: strategic direction; innovation; competitiveness; MSEs; administrative management. 


\section{INTRODUCCION}

En el mundo de las Pymes, la innovación tecnológica, surge cómo una estrategia en respuesta a los desafíos impuestos por la globalización de la economía, por los avances tecnológicos y la expansión del uso de nuevas herramientas en información y comunicaciones. Cuando se habla de innovación tecnológica en el mundo empresarial, hay que hacer referencia a las mejoras en productos o servicios que ya existen en el mercado, introducir productos que ofrezcan algún plus o valor agregado, actualizar los sistemas de información, renovar herramientas tecnológicas o maquinarías y equipos que permitan aumentar la productividad en las empresas, la gestión de cambios en organización y administración de las empresas (Riascos et al., 2016). Dadas las políticas estatales que apoyan las ideas de emprendimiento y creación de pequeñas y medianas empresas, cómo un eslabón de gran importancia en la economía actual del país, ya que estas se constituyen en fuente de generación del $67 \%$ del empleo en el territorio nacional y que las pymes corresponden al 99,6\% de las empresas registradas en el Registro único Empresarial -Rues(Confecámaras, 2016), ha surgido la necesidad de analizar el comportamiento de estas empresas, en cuanto a temas cómo la supervivencia de las pymes en el mercado y los motivos que coadyuvan en el éxito o fracaso de su permanencia en el tiempo; es ahí donde se encuentran a la innovación tecnológica y al direccionamiento estratégico cómo factores determinantes esta ecuación.

La ciudad de Barranquilla es considerada como la más importante en la región caribe, y ha sido protagonista en las últimas décadas de un crecimiento económico gracias su ubicación geográfica, a la facilidad de acceso al océano atlántico y al rio Magdalena se ha convertido en Distrito Especial Industrial y Portuario, encontrando en ella diversidad en cuanto a los sectores de la economía, principalmente en el logístico, comercial, de servicios, metalmecánico y de la construcción (Velandia et al., 2016); repuntando en exportaciones y actividades de comercio exterior, la convierten en el punto propicio para aplicar estrategias con enfoques de direccionamiento empresarial, que propendan al desarrollo de nuevos procesos y productos, que posibiliten la adaptación a la nueva tecnología y promuevan la incorporación de prácticas gerenciales en los aspectos organizacionales de todo ente productivo. Lo anterior, se realiza para favorecer la productividad y competitividad, ponderando su posición en el mercado (Thorsten et al., 2013; Prescott, 2016). De esta manera, la gestión tecnológica surge como respuesta a la carencia de dinámicas innovadoras en la Pymes, que se reflejan en la baja calidad en productos y prestación de servicios, en el alto costo de los productos y a la necesidad de manejar el factor tecnológico como una estrategia competitiva, para responder al ritmo con el cual las tecnologías entran al mercado y para enfrentar los desafíos de los agentes de cambios (Wilder, 2011; Tarapueza et al., 2016).

La planificación estratégica y el direccionamiento estratégico son enfoques que presentan variedad de opciones o esquemas; la dirección estratégica es un concepto más general que puede abarcar al primero. Ansoff (1997), establece que un resultado final de la dirección estratégica es el potencial para el logro futuro de los objetivos de la organización. Aunque no se puede negar la importancia de las áreas de dirección operativa, es vital reconocer que el alcance de la dirección estratégica; es mayor que el de cualquiera de las áreas de dirección operativa (Bustamante et al., 2017). El objetivo principal de la dirección estratégica es formular las estrategias ajustadas al requerimiento de los mercados y orientar los planes de actuación empresarial, intentando anticiparse a previsibles acontecimientos futuros que afecten o impacten a la organización (Arévalo et al., 2017; Álvarez y Bernal, 2017).

El direccionamiento estratégico, lo integran los principios corporativos ampliamente conocidos como: visión y misión. Los principios corporativos son el conjunto de valores, creencias, normas, que regulan la vida de una organización y que estimulan en ella el cumplimiento de unas proyecciones preconcebidas. Ellos definen aspectos que son importantes para la organización y que deben ser compartidos por todos. Por tanto, constituyen la norma de vida corporativa y el soporte de la cultura organizacional que abona el terreno para garantizar unos resultados, además de buscar que aquellos que conforman las organizaciones tengan oportunidades de desarrollo y emprendimiento que finalmente generar valor para todos (Quevedo y Rodríguez, 2008; Del Río, 2012; Salas et al., 2016).

La innovación tecnológica se define como la aplicación de la ciencia y la tecnología en una nueva dirección, seguida de un éxito comercial, en un mercado o aparato económico donde la competencia es vital. (OCDE, 1996). Esta competencia brinda a la empresa un factor diferencial conocido como inteligencia corporativa (Hernández et al., 2015). La innovación tecnológica se configura en el desarrollo de nuevos productos y procesos que presentan cambios significativos, y adquiere valor cuando esta innovación propuesta es aceptada por el mercado y por los usuarios y consumidores que lo componen (innovación de productos) o se incorpora en el diseño de los procesos de producción (innovación de procesos), (Yéndez, 2000). Esta nueva corriente, viene a responder a una serie de requerimientos, que las economías mundiales han generado en procura del cumplimiento de las nuevas demandas de desarrollo económico (Niebles et al., 2016; Ben-Hdech et al., 2015). 
La innovación es una habilidad competitiva para las organizaciones que logran combinar y articular aspectos de la cultura, la organización empresarial, la investigación y el desarrollo, la transferencia de tecnología acompañada de un enfoque hacia la especialización y por supuesto la orientación motivadora hacia el talento humano como impulsor o dinamizador de los cambios (Simanca et al., 2016; Khalid, 2015). Las prácticas innovadoras en las organizaciones conducen a generar las ventajas competitivas sostenibles. Como estrategia de desarrollo empresarial, la innovación no sólo está orientada a la generación de nuevos productos y procesos, sino también, a la adaptación y mejora de tecnologías y a la adopción de cambios en la cultura empresarial, en fin, a la introducción permanente de cambios que permitan incrementar la productividad y competitividad de las empresas (Tapias, 2000; Balza,2016). Por lo tanto, el objetivo de este trabajo es evaluar el desempeño innovador de diez pymes ubicadas en la ciudad de Barranquilla, Colombia. Mediante esta investigación se identificará el monto de inversión y los tipos de innovación que las empresas realizan a su organización.

En la actualidad, las exigencias en competitividad se encuentran en un nivel bastante elevado, teniendo en cuenta que la globalización de la economía. Ante esta realidad, se hace necesario que las empresas fortalezcan sus ventajas competitivas y obtengan sostenibilidad a fin de adaptarse y subsistir en un mercado complejo, como lo afirma Zuleta (2011) en resultados a entrevistas realizadas a empresas del sector privado que prestan servicios de financiamiento a las Pymes en Colombia, estas prevén en un corto plazo, que presentaran un aumento considerable en productos dirigidos a este clase de empresas, cómo manejo integral de tesorerías, operaciones fiduciarias, operaciones de comercio exterior, cuentas electrónicas a través de internet, operaciones de cobertura cambiaria y mesas de dinero especializadas para Pymes, dicha proyección, es una muestra de la conveniencia de la innovación en el crecimiento de estas empresas. Para cumplir con este objetivo, se pueden aplicar diversos enfoques que van desde lo administrativo hasta la innovación tecnológica (Echeverry y Trujillo, 2012). El enfoque tecnológico, ofrece multivariedad de herramientas que pueden estimular la función organizacional y procrear un nuevo escenario para el desarrollo sostenible (Mojica et al., 2015; Mahdzur y Salim, 2015).

\section{MATERIALES Y MÉTODOS}

Esta investigación fue de tipo descriptivo, para el desarrollo de la investigación se evaluaron 10 empresas de la ciudad de Barranquilla, Colombia. Categorizadas como pequeñas y medianas empresas (pymes), según lo establecido por la ley 905 del 2004, se aplicó un instrumento elaborado por los investigadores, con el fin de identificar primero la categoría de la empresa y segundo el monto de inversión, tipo y enfoque de la innovación. Las 10 empresas seleccionadas según su registro mercantil se clasifican así: 6 empresas de servicios con los códigos CIIU 4742 y 4 empresas de corte industrial con los códigos CIIU 2822 . El instrumento fue dirigido a las personas líderes o responsables de la dirección.

Las variables utilizadas fueron: número de trabajadores, actividades innovadoras desarrolladas por las empresas, monto de inversión, ámbito de enfoque de las innovaciones e inclusión de las tecnologías en la misión y visión. Los datos fueron tabulados en el programa Microsoft Excel en donde se analizaron los resultados arrojados, se identificaron las posibles causas y se establecieron posibles soluciones. Seguidamente y en base a un cálculo inferencial, se logró establecer la correlación derivada entre el crecimiento económico y la adopción de políticas de direccionamiento estratégico e innovación, para medir el impacto de estas variables en el entorno económico de las organizaciones.

\section{RESULTADOS}

Las empresas objeto de este estudio, se localizan en un $60 \%$ en el sector industrial y un $40 \%$ en el sector servicios, Además se categorizan de acuerdo al número de empleados y activos, según la Cámara de Comercio de Barranquilla (ver figura 1), se observa que un $60 \%$ se categorizan como medianas empresas, mientras que el $40 \%$ restante, se ubican como pequeñas empresas, lo que las constituye como pymes.

Al observar los resultados de la figura 1, se encuentra que un $60 \%$ de las empresas, se clasifican cómo medianas por tener entre 51 y 200 trabajadores empleados, mientras que el $40 \%$ de las empresas restantes, pertenecientes a la muestra, pertenecen a las pequeñas empresas teniendo entre 11 y 50 empleados; que en comparación con lo arrojado por la investigación de Rico et al. (2015), las microempresas corresponden a un $32 \%$, las pequeñas empresas a un $44 \%$, las medianas y grandes empresas aportan un $12 \%$ cada una en esta categoría.

En la figura 2, se observa que un $80 \%$ y $90 \%$ de las empresas implementan actividades de comercialización y diseño respectivamente, mientras que un $80 \%$ no realiza cambios organizacionales ni adquieren tecnologías no incorporadas al capital, cabe destacar que solo un $40 \%$ de las empresas implementan actividades enfocadas a la innovación y desarrollo. Lo anterior refleja de que las empresas y organizaciones no se enfocan completamente en la adquisición de tecnologías e innovaciones. 


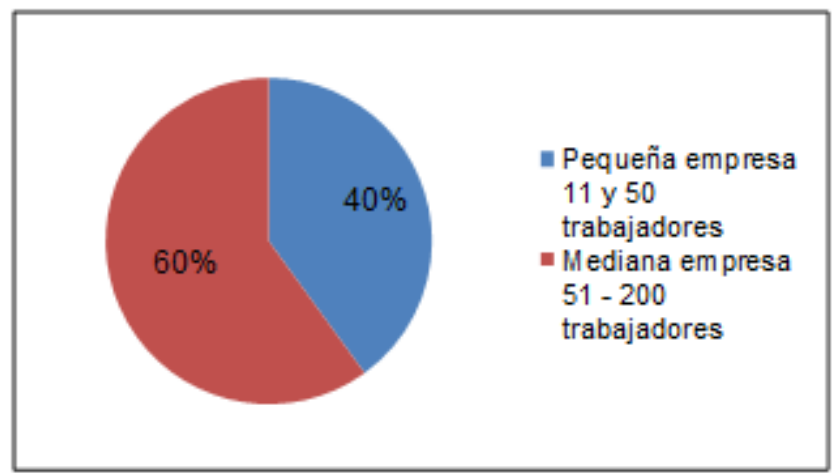

Fig.1: Categorización de las empresas

En la figura 2, se puede observar que el $90 \%$ de las empresas incluidas en esta muestra, prefieren implementar actividades innovadoras de diseño, mientras que un $80 \%$, manejan actividades de innovación en comercialización, el tercer lugar con un $40 \%$ lo ejercen empresas que emplean actividades de innovación y desarrollo, adquiriendo tecnología incorporada al capital, y un $20 \%$ de ellas, adoptan cambios organizacionales, dejando en un último lugar con un 10\% la opción de la capacitación cómo actividad innovadora, mientras que Rico et al. (2015), afirman que el $60 \%$ de las empresas en Barranquilla, están de totalmente de acuerdo en implementar la innovación en sus políticas, y solo un $4 \%$ no realiza ningún tipo de actividad innovadora.

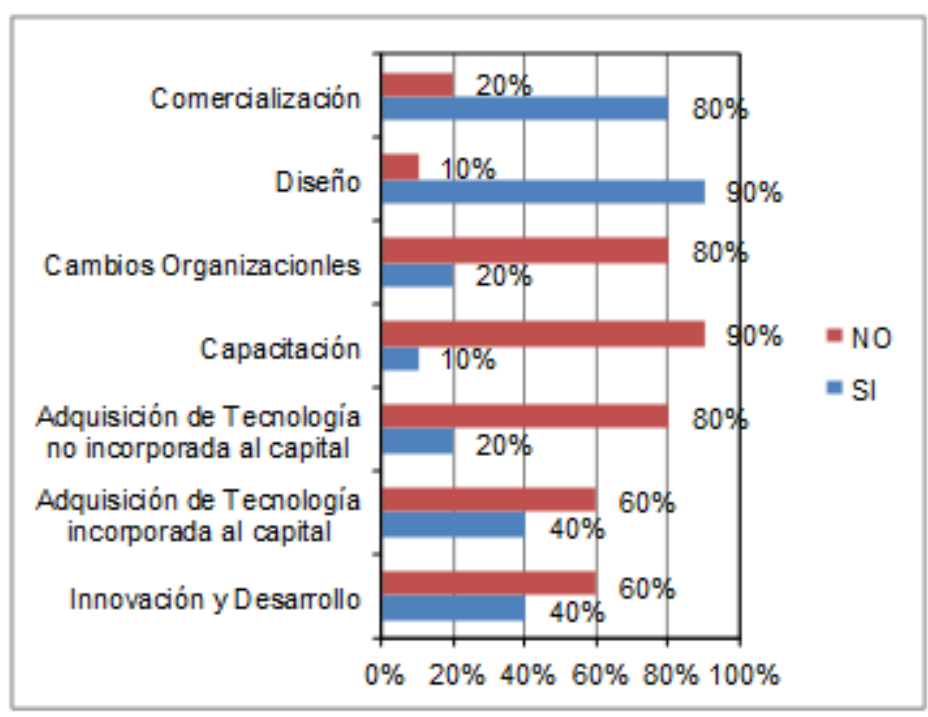

Fig. 2: Actividades Innovadoras

En la figura 3 se observa que solo un 40\% de las empresas invirtió en los últimos cinco años de 300 a 600 millones de pesos en cambios tecnológicos en donde estén implicados productos y procesos nuevos, se concluye que las organizaciones destinan un bajo porcentaje de sus ingresos en la inversión de cambios para la mejora de sus servicios. En la figura 3, se halla que el $40 \%$ de las empresas pertenecientes a la muestra invierte entre 300 y 600 millones de pesos en actividades de cambio tecnológico, otro $30 \%$, destina un rubro de entre 100 y 300 millones de pesos para este fin, y el otro 30\% emplea entre 600 y 1200 millones de pesos para el logro de este objetivo.

Se observa en la figura 4 , que un $40 \%$ de las empresas han enfocado su ámbito de innovación hacia el desarrollo organizacional, cabe destacar cualquier estrategia de crecimiento o diversificación implica cambios en la estructura organizacional y estos facilitan la implementación de la innovación. Se identifica que solo un $40 \%$ de las empresas incluyen en su misión y visión las innovaciones tecnológicas con las que trabaja su organización, mientras que un $60 \%$, no da a conocer que tipos de tecnologías implementa.

Dentro de los resultados encontrados en la figura 4, se encuentra que el $40 \%$ de las empresas encuestadas, han enfocado su ámbito de innovación hacía el desarrollo organizacional, un $30 \%$ se enfoca en el ámbito de los procesos, otro $20 \%$ se encauza al ámbito de los productos y el $10 \%$ restante, orienta sus esfuerzos en el ámbito de las operaciones, entre tanto Rico et al. (2015), afirman que un $84 \%$, destina una buena parte de su presupuesto para invertir en innovación tecnológica, otro $8 \%$ de la empresas, no dispone de un presupuesto para este fin y el $8 \%$ restante le es indiferente el tema de la inversión en innovación tecnológica. 


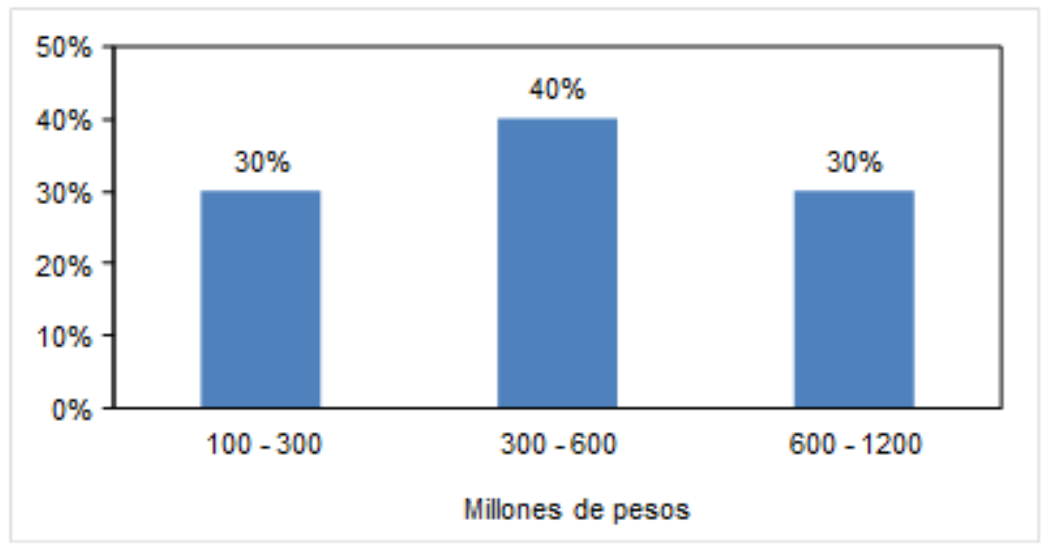

Fig. 3: Inversiones en cambio tecnológico

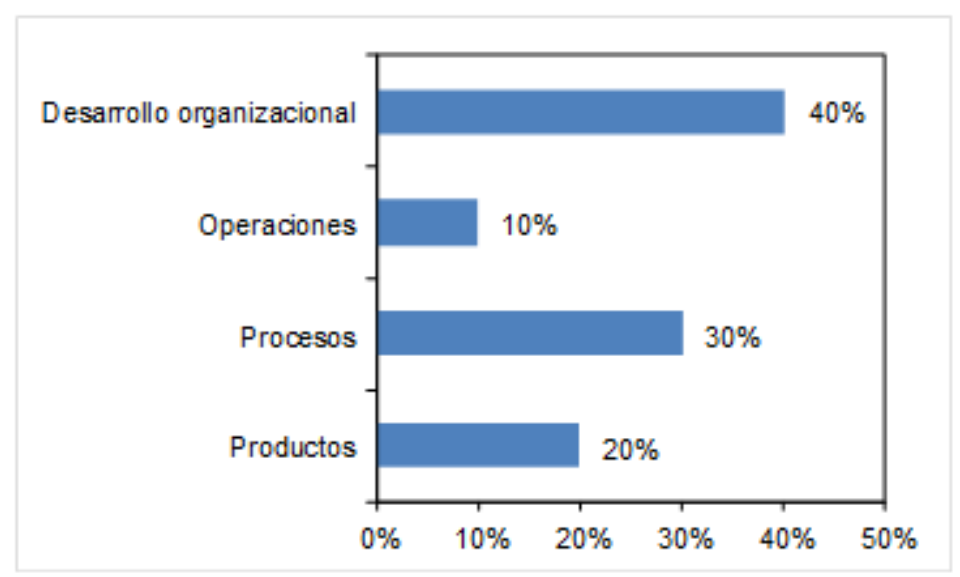

Fig. 4: Ámbito de las innovaciones.

Para profundizar en los hallazgos encontrados, se aplicará paralelamente, un análisis de correlación que, es la forma numérica en la que la estadística ha podido evaluar la relación de dos o más variables, es decir, mide la dependencia de una variable con respecto de otra variable independiente (Jurado, 2011). Por lo citado anteriormente, se logró determinar que las variables de direccionamiento estratégico/innovación y crecimiento económico, están directamente vinculadas; al indagar sobre la política de inversión en esta temática, los resultados tabulados en Excel, muestran que a mayor inversión hubo un crecimiento organizacional y productivo superior. A continuación, en tabla 1 se analizan las correlaciones.

Tabla 1: Correlación entre Direccionamiento Estratégico/Innovación y Crecimiento Económico

\begin{tabular}{l|c|c|c|c|c|c|}
\cline { 2 - 7 } & $f$ & $x$ & $y$ & $x^{2}$ & $y^{2}$ & $x^{*} y$ \\
\cline { 2 - 7 } Empresa 1 & 1 & 10 & 2 & 100 & 4 & 20 \\
\cline { 2 - 7 } Empresa 2 & 1 & 25 & 5 & 625 & 25 & 125 \\
\cline { 2 - 7 } Empresa 3 & 1 & 15 & 3 & 225 & 9 & 45 \\
\cline { 2 - 7 } Empresa 4 & 1 & 12 & 1 & 144 & 1 & 12 \\
\cline { 2 - 7 } Empresa 5 & 1 & 35 & 8 & 1225 & 64 & 280 \\
\cline { 2 - 7 } Empresa 6 & 1 & 12 & 2 & 144 & 4 & 24 \\
\cline { 2 - 7 } Empresa 7 & 1 & 8 & 4 & 64 & 16 & 32 \\
\cline { 2 - 7 } Empresa 8 & 1 & 7 & 1 & 49 & 1 & 7 \\
\cline { 2 - 7 } Empresa 9 & 1 & 14 & 3 & 196 & 9 & 42 \\
\cline { 2 - 7 } Empresa 10 & 1 & 2 & -5 & 4 & 25 & -10 \\
\cline { 2 - 7 } & $\mathbf{1 0}$ & $\mathbf{1 4 0}$ & $\mathbf{2 4}$ & $\mathbf{2 7 7 6}$ & $\mathbf{1 5 8}$ & $\mathbf{5 7 7}$ \\
\cline { 2 - 6 } & & \multicolumn{5}{|c}{}
\end{tabular}


Tabla 1 (continuación)

Media Arit. $\quad 14,00 \quad 2,40$

Varianza $\quad 9,0333 \quad 3,1686$

\begin{tabular}{|l|l|l|}
\hline Covarianza & 24,10 & 24,10 \\
\hline
\end{tabular}$\quad$ Direccionamiento / Innovación

\begin{tabular}{|l|l|l|l|l|l|}
\hline Correlación & 0,8420 & 0,8420 & Y Crecimiento Económico
\end{tabular}

En la variable $X$ expresada en millones, se alimentaron los valores aproximados de inversión del último año en lo referente a innovación y direccionamiento estratégico, seguidamente, en la variable $Y$, se ubicó la utilidad estimada y cuantificada en millones que representó el crecimiento económico, que estas empresas obtuvieron gracias a la inclusión de las políticas citadas. Las escalas valorativas de referencia, que autores como Jurado (2011), proponen para este tipo de análisis son las que siguen (Tabla 2):

Tabla 2: Escala valorativa de referencia propuesta por Jurado (2011)

\begin{tabular}{|l|l|}
\hline$r<0,20$ & Leve \\
\hline $0,21<r<0,40$ & Baja \\
\hline $0,41<r<0,70$ & Moderada \\
\hline $0,71<r<0,90$ & Alta \\
\hline $0,91<r<1$ & Muy Alta \\
\hline$r=1$ ó -1 & Perfecta \\
\hline
\end{tabular}

Al contrastar el resultado alcanzado de 0.8420 , se concluye que la relación entre inversión en innovación y políticas de direccionamiento estratégico con el crecimiento económico, se ubica en la escala Muy Alta. Este consolidado, permite establecer la necesidad y pertinencia de promover en las organizaciones actuales, la inclusión de las mismas en sus planes de desarrollo, garantizando así que, a medida que la inversión en políticas estratégicas aumente, crecerá también la rentabilidad y demás objetivos corporativos propuestos, coincidiendo con Rico et al. (2015), quienes afirman que un $90 \%$ de las empresas, maneja dentro de su direccionamiento estratégico, la cultura de la innovación tecnológica, mientras que solo un $4 \%$ no lo tiene dentro de sus lineamientos.

\section{CONCLUSIONES}

A partir del trabajo se evidencia que en cada una de las etapas del proceso de direccionamiento estratégico se debe establecer una adecuada integración de la organización con el entorno, tomando como base de su cambio y mejora continua, la innovación tecnológica y la gestión administrativa. Solo así, se puede lograr un avance sustancial que corresponda con las exigencias del sistema o entorno.

Para ello las empresas, deben enfocarse en invertir económicamente en la implementación de innovaciones, que se ajusten a su perfil y que contribuyan al crecimiento de su organización, ya que la correlación entre ambas variables es supremamente alta y en este caso es positiva. Sus innovaciones deben enfocarse en reforzar las áreas o servicios que más deficiencia presente, ya que al identificarlas y mejorarlas les permitiría disminuir los riesgos y mantener la mejora continua de su organización.

De igual manera es importante que las organizaciones incluyan en su visión y misión las innovaciones tecnológicas que utilizan para que el cliente tenga conocimiento de los recursos que utiliza la organización para el desempeño de sus servicios, esto le brinda al usuario más confiabilidad y credibilidad hacia la empresa. Mientras que estas al implementar nuevas tecnologías aumentan su competitividad y asegura su crecimiento como organización en el mercado y evitan convertirse en organizaciones obsoletas.

Por esta razón, las empresas deben ejecutar sus decisiones de forma contundente, para responder a los cambios del entorno tecnológico, entre las cuales se pueden destacar: la practicidad de los procesos, el uso integral de los sistemas de comunicación, la inversión en actividades de investigación y el desarrollo de una cultura de conocimiento, que permita la innovación constante con enfoque en la productividad de alto nivel. 


\section{AGRADECIMIENTOS}

Este artículo es el resultado de investigación del Proyecto: "Impacto de los sistemas integrados de gestión en las pymes de la región Caribe", desarrollado con aval de la Corporación Universitaria Latinoamericana (CUL), y la colaboración de los grupos de investigación GIIO y Denys Cervantes.

\section{REFERENCIAS}

Álvarez, E. y Bernal, C., Open Innovation Model: Focus on Human Potential, doi: 10.4067/S071807642017000100007, Journal of Theoretical and Applied Information Technology, 28(1), 65-76 (2017)

Ansoff, H., La dirección y su actitud ante el entorno, 1르 Ed., 77-80, Ediciones Deusto, Bilbao, España (1997)

Arévalo, D., Padilla, C., Lozano, M., Bustamante, M. y Vidal, C., Contrasting the Business Productivity Paradox of the use of Information Technology: the Ecuadorian Case, Inf. Tecnológica, 28(1), 172-175 (2017)

Balza-Franco, V., Economías de Aglomeración Empresarial y Políticas Públicas de Competitividad desde un Enfoque Global hacia un Contexto Latinoamericano: Una Revisión Conceptual, Rev. Espacios, 37(36), 8 (2016)

Ben-Hdech, A., Ghanou, Y. y El Qadi, A., A new algorithm for optimizing the selforganizing map, Journal of Theoretical and Applied Information Technology, 78(3), 415-419 (2015)

Bustamante, E., Zúñiga, A. y Toro, I., Design of a strategic management plan for the fishery sector, Revista Espacio, 38(21), 18 (2017)

Confecámaras, Nacimiento y Supervivencia de las Empresas en Colombia, https://goo.gl/LMrWpb, Análisis Económico, 11, 11-12 (2016)

Del Río-Cortina, J., Desarrollo de Actividades de Emprendimiento con Innovación y Responsabilidad Social en los Hoteles en la Ciudad de Cartagena de Indias, 7(2), 113-124 (2012)

Echeverry, C. M. y Trujillo, M. L., La Gestión del Conocimiento en las PYMES de Colombia, Revista Virtual Universidad Católica del Norte, 38(1), 159 (2012)

Hernández, H., G., Muñoz, D. y Jiménez, A., Gestión de la Información Empresarial en las Organizaciones Inteligente, Revista Escenarios, 12(4), 40 (2015)

Jurado, Y., Metodología de la Investigación, 5ª Ed., Reimpresión, 35-75, Esfinge, Mexico (2011)

Khalid, A., Implementing Electronic Documentation Management System, Journal of Theoretical and Applied Information Technology, 77(1), 52-55 (2015)

Mahdzur, S. y Salim, J., Information systems integration factors in organization: towards government information systems sustainability, J. of Theoretical and Applied Information Technology, 71(2), 237-242 (2015)

Mojica, J., Barandica, A., Rodero, L., Franco, M., Hernández, H. y Arboleda, S., La Función Administrativa en la Era de las TIC, Revista Lasallista de Investigación, 12(2), 139-151 (2015)

Niebles, W.A., Hernández, H.G. y Cardona, D.C., Gestión Tecnológica del Conocimiento: Herramienta Moderna para la Gerencia de Instituciones Educativas, doi: 10.19053/20278306, Revista de Investigación Desarrollo e Innovación, 7(1), 25-36 (2016)

OCDE, La Innovación Tecnológica: Definiciones y Elementos de Base, Redalyc, 3(6) 133 (1996)

Prescott, M., Big Data: Innovation and Competitive Advantage in an Information Media Analytics Company, Journal of Innovation Management, 4(1), 92-113 (2016)

Quevedo, D. y Rodríguez, F., Direccionamiento Estratégico para la Empresa Mavitours Transportes Especiales S.A., Universidad de la Salle - Bogotá, 1, 57 (2008) 
Riascos, S., Aguilera, A. y Armando, H., Investment in Information Technology and Communications and its relationship with the strategic direction for SMEs in Cali - Colombia, Revista Ibérica de Sistemas e Tecnologias de Informação, 18(6) 7-13 (2016)

Rico, H., Martínez, Z., Rico, F., Amar, P., Comportamiento Innovador del Sector Alimentos en el Distrito de Barranquilla, https://goo.gl/XPrzK2, ISBN: 978-958-8930-06-0, pp. 45-56, Ediciones Simón Bolívar, Barranquilla-Colombia (2015)

Salas, K., Acosta, C., Sandoval, L., Pacheco, G. y Mercado, M., Strategic analysis of cluster of logistics services, https://goo.gl/DFgBLc, ISSN: 0798-1015, Revista Espacios, 37(28) 3-8 (2016)

Simanca, M., Montoya, L. y Bernal, C., Gestión del Conocimiento en Cadenas Productivas. El Caso de la Cadena Láctea en Colombia, Revista Información Tecnológica, 27(3), 93-106 (2016)

Tapias, H., Gestión Tecnológica y Desarrollo Tecnológico, Fac. de Ingenieria U. de Antioquia, 159-160 (2000)

Tarapueza, E., Guzmán, B. y Parra, R., Strategy and innovation in Colombian MSMEs winning the Innova award 2010-2013, ISSN: 0123-5923, Estudios Gerenciales, 32(1), 170-180 (2016)

Thorsten, K., Todd D. y Thomas B., Creating a sustainable innovation environment within large enterprises: a case study on a professional services firm, Journal of Innovation Management, 1(1), 55-84 (2013)

Velandia, G., Hernández, L., Portillo, R., Alvear, L., Crissien, T., Rasgos de la Administración de la Microempresa en Barranquilla, Colombia, https://goo.gl/QZ7RdH, ISSN: 07981015, Revista Espacios, 37(9), 7 (2016)

Wilder, J., Orozco, G., Vergara, J. y Martínez, D., Diagnóstico de Estrategia de Innovación en Grupos de Investigación, https://goo.gl/oraqTx, ISSN: 0718-2724, Journal of Technology Management \& Innovation, 6(3), 197-200 (2011)

Yéndez, N. E., La Innovacion Tecnologica, Revista Medisan, 4(4), 2 (2000)

Zuleta, L. A., Documento de Proyecto: Política Pública e Instrumentos de Financiamiento a las Pymes en Colombia, Comisión Económica para América Latina y el Caribe (CEPAL), https://goo.gl/ruV1R5, 37-38, Copyright @ Naciones Unidas, Impreso en Santiago de Chile, julio (2011) 